of date. Our aim was to develop a new informant-based IADL questionnaire. The purpose of this questionnaire is to measure subtle deficits in IADL and to be helpful in diagnosing early dementia. The questionnaire also had to be suitable for dementia patients younger than 65 years of age. Methods: We defined the construct IADL by interviewing experts. All IADL items of existing IADL questionnaires were inventoried. Twelve experts, including neurologists and occupational therapists, were asked to select items sensitive for early dementia and to generate new items. Interviews with 30 informants were performed to further select and generate new items. After this selection, experts and informants were asked to judge the interpretability of the items in order to select unambiguous items. A pilot study was conducted to test the practicability of the questionnaire. Results: Of the 139 items from existing IADL questionnaires, experts selected 57 items. Sixty-four items were newly suggested, leading to a total of 121 items. Of these 121 items, 36 were marked as ambiguous by informants and experts. After the removal of these items, the questionnaire consisted of 75 items. The pilot study with informants resulted in negative feedback on the complexity of the questionnaire. We therefore computerized the questionnaire to an internet-based format. Using this format, items were tailored to the individual responses, thus greatly reducing the complexity of the questionnaire. The experiences of 118 informants demonstrate a practicable and easy to complete questionnaire. Preliminary results on the reliability and validity will be presented. Conclusions: There is a need for a new IADL questionnaire, applicable for early dementia and for early-onset dementia. The Amsterdam IADL questionnaire serves both purposes and preliminary results show that this questionnaire is a promising new tool in the evaluation of dementia.

\section{F3-01-02 MEASUREMENT ISSUES IN TRACKING THE ONSET OF ALZHEIMER'S IN PROBABILITY SURVEYS: COMPARING THE HRS AND THE NLTCS}

Martha I. Sayre, James W. McNally, University of Michigan, Ann Arbor, MI,USA.Contact e-mail: msayre@umich.edu

Background: Increasingly, longitudinal studies on the aging lifecourse seek to measure a multitude of factors, from health to family structure to economic stability. Alzheimer's remains a relatively rare outcome in the general population, capturing individuals in surveys prior to their being diagnosed with the disease is equally rare. Even with large sample populations, the focus and design of a survey impacts the likelihood of following individuals with cognitive impairments through time and the types of questions asked regarding cognitive functioning. It is presently unknown if these differences impact observed results in a statistically significant manner. Methods: We compare two highly regarded gerontological surveys; The Health and Retirement Survey (HRS) and the National Long Term Care Survey (NLTCS). Both studies have multiple waves of data collection and both have large sample sizes to follow small populations through health transitions. The HRS focuses primarily on the economic stability and health of the elderly while the NLTCS focuses primarily on health transitions, following respondents even if they enter institutionalized care. This paper looks at similar populations in both studies and compares the consistency of results across the two studies. Results: Both studies offer insight into the health and socioeconomic characteristics of their respondents who become cognitively impaired across the studies. The NLTCS provides much finer detail on the health related process, testing and health transitions, while the HRS offers greater insight into the impacts of spousal support by interviewing partners. Both studies offer insights but the results are not directly comparable. Conclusions: The NLTCS was designed to capture the onset of disability and its stronger health focus offers more opportunities for the in-depth examination of both the transitional process from healthy to cognitively impaired, up to and including institutionalization. The HRS began with a relatively weak battery of cognitive tests that it has improved in subsequent waves, now add biomedical specimen collection. The value of the NLTCS is somewhat weakened by its five year intervals between measurement periods making the identification of transitions difficult. Like the HRS, these issues are being addressed in the form of an undated survey, the NHAT which enters the field in 2010 .

\section{F3-01-03 FITNESS-TO-DRIVE IN OLDER ADULTS WITH DEMENTIA}

David B. Carr ${ }^{1}$, Peggy Barco ${ }^{1}$, James C. Galvin ${ }^{1}$, Brian R. Ott ${ }^{2}$, John C. Morris ${ }^{1},{ }^{1}$ Washington University School of Medicine, St. Louis, MO, USA; ${ }^{2}$ Warren Alpert Medical School of Brown University, Providence, RI, USA.Contacte-mail:dcarr@im.wustl.edu

Background: Performance-based road tests are considered the gold standard for measuring driving fitness, yet these examinations are nonstandardized, expensive, often unavailable, and have safety risks. Thus, other reliable and valid office or outpatient tools are needed to determine who is safe to drive. Methods: To compare informant-based questionnaires and brief cognitive tests of individuals with dementia with three dichotomous outcome measures: a performance-based road test (a modified Washington University Road Test), motor vehicle crashes as reported by the informant in the past year, and an overall rating of the quality of the driver by an informant. All participants had a diagnosis of dementia as judged by a physician, a physician referral for a driving assessment, a positive score of $>2$ on the AD- 8 dementia screening interview, and an active drivers license. The primary predictor measures were the newly developed Driving Behaviors Questionnaire (DBQ), the Functional Assessment Questionnaire (FAQ), the AD-8, and brief cognitive measures (Clock Drawing Test-Freund (CDT-F), Trailmaking Test (parts A and B). Results: 102 participants met inclusion criteria for the study. $65 \%$ failed the road test, $20 \%$ experienced a motor vehicle crash in the past year, and 35\% were rated as impaired drivers by their informant. Scores for all measures were significantly more impaired for drivers who failed the road test or were given an impaired driving rating by the informant. ROC curves showed good ability for these tools to individually discriminate road test performance $(\mathrm{AUC}=.71-.78)$. Data reduction via factor analyses explained $68 \%$ of the variance and included constructs of informant assessments, executive ability, psychomotor speed, and attention/construction. Logistic regression models for predicting failure on the road test included; the AD-8 (OR 1.4), the CDT-F (OR 1.6), and Trailmaking B (OR1.0) which correctly classified $78 \%$ of cases. The estimated time to complete the tasks was 6 minutes. Conclusions: Informant questionnaires assessing functional abilities, in addition to tests of attention, psychomotor speed and visuospatial skills, may provide a useful and brief in-office assessment of driving safety for clinicians.

\section{F3-01-04 CAREGIVER WELLBEING: A LONGITUDINAL PERSPECTIVE ON RELINQUISHMENT AND GRIEF}

Lynsey J. Brown, Malcolm J. Bond, Flinders University, Adelaide, South Australia, Australia.Contact e-mail: lynsey.brown@flinders.edu.au

Background: Older Australians who experience dementia are commonly assisted by family members, such as spouses, who provide support as primary caregivers. Research consistently demonstrates a tendency for caregivers to report a decline in physical and psychological wellbeing as their time in the role continues. While there is some support for the notion that wellbeing will improve post-relinquishment, there have been other suggestions that the chronic stress of caregiving may be too damaging to be appeased by cessation of the role. It seems plausible that the manner in which caregivers transition out of the role (due to their care recipient's move into permanent residential accommodation or the death of their care recipient) will affect both grief and wellbeing. Methods: With much of the evidence around wellbeing having been derived from cross-sectional research, there remains a need to explore the changes experienced by caregivers through the application of longitudinal methods. The current quantitative study entailed six home visits (spanning seven years) during which participants were assisted in completing a questionnaire that included measures of physical and psychosocial wellbeing, and dementia severity. Visits were initially conducted with 200 individuals who nominated themselves as primary caregivers to a co-resident spouse diagnosed with dementia, and 60 community members (age and sex matched to caregiver sample). Caregivers who transitioned out of their role during the study were retained as either relinquished (spouse in residential care) or bereaved caregivers (spouse deceased). Results: There is a trend toward bereaved caregivers showing improvements in wellbeing 